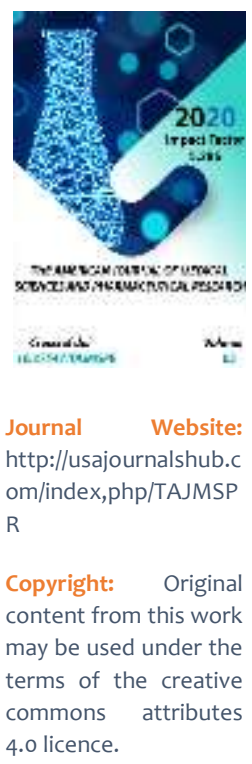

\title{
Experience In Surgical Treatment Of Diaphysical Fractures Of The Shin Bones
}

\author{
Mizrapov F.A. \\ Samarkand State Medical Institute, Uzbekistan
}

\section{ABSTRACT}

The results of blocked intramedullary osteosynthesis (BIOS) in 50 patients with fractures of the distal and diaphysis of the tibia are presented. Blocked intramedullary osteosynthesis is a reliable and predictable method for the treatment of diaphyseal fractures of the tibia, which allows the restoration of limb function in the shortest possible time in most patients.

\section{KEYWORDS}

Blocking intramedullary osteosynthesis, tibia, implant.

\section{INTRODUCTION}

Fractures of the shin bones occupy, according to various sources, the first place, accounting for 13 to $21.4 \%$ of all injuries of the musculoskeletal system $[1,2]$. The incidence of diaphyseal tibial fractures is $26-32$ cases per 100,000 population per year. The treatment and early recovery to work of patients with unstable diaphyseal fractures of the shin bones are still far from being solved and the most urgent problem. The difficulty of treating patients with unstable leg fractures, the complexity of their reduction and the impossibility of stable fixation often leads to an unsatisfactory treatment outcome, which, as a rule, increases the disability of patients $[1,3]$.

In the treatment of diaphyseal fractures of the lower leg bones, various methods of surgical 
treatment are used. There is no specific standard of treatment for these fractures. Different researchers have different opinions when choosing a treatment method. In modern traumatology, when choosing a treatment method, preference is given to the one that provides a quick restoration of the limb function, as close as possible to physiological $[4,5]$. While some authors argue that transosseous osteosynthesis is an effective method in the treatment of patients with fractures of the shin bones, others prefer intraosseous and extraosseous methods of fixing the fracture.

Interlocked intramedullary osteosynthesis has become the standard treatment of most diaphyseal fractures of the lower extremities in developed countries. The biomechanical features of the method (the location of the fixator in the center of the bone) and low invasiveness, with the correct technical execution and appropriate material support, make it possible to achieve a quick predictable recovery of the function of the injured segment with a minimum number of complications. The shortage and high cost of implants, insufficient technical equipment and the need for special training of traumatologists for a long time held back the introduction of this method $[1,6,7]$.

\section{PURPOSE OF THE STUDY}

To study the results of intramedullary blocking osteosynthesis in diaphyseal fractures of the shin bones.

\section{MATERIALS AND METHODS}

Intramedullary osteosynthesis of the tibia with blocking has been used in the Samarkand branch of the Republican Scientific Practical Medical Center of Traumatology and Orthopedics since 2017. This study included 50 patients with a follow-up period of at least 12 months after primary intramedullary osteosynthesis of fractures. ChM (Poland) implants were used in 46 patients and in 4 SIGN (USA) patients. The average age of the patients was 45 years (range 20 to 71 ). The average duration of the intervention after the injury was 12 days, with 16 patients operated on the day of admission, 9 patients within the period up to 3 days from the moment of injury, 5 - from 4 to 7 days, 12 - in the second week, 8 - in the third or fourth weeks after injury.

Technique for performing interventions. All operations were performed with the patient supine using general or spinal anesthesia. A healthy limb was placed on an abduction support, and an injured limb was placed on a support in the lower third of the thigh with a freely hanging tibia, while providing a flexion position in the knee joint of about 1000. Access to the insertion point was through the patella's own ligament with its longitudinal fibration. After opening the medullary canal in 38 (76\%) cases, it was reamed with hand drills or drills with a mechanical drive along the guide wire. The canal was processed sparingly, with an emphasis on the tapering zone to determine the most suitable retainer diameter $(1-2 \mathrm{~mm}$ less than the drill diameter). In 12 (24\%) cases, reaming of the medullary canal was not performed. Fracture reduction was always performed manually without the use of additional devices. In 46 cases, the reduction and insertion of the rod were performed, closed, in 4 cases, minimal approaches were performed (1 case - segmental fracture, 1 - long co-spiral fracture, 2 - after the injury was 15 and 24 days old). To correct angular deviations of short periarticular fragments of the proximal part of the tibia, in 5 cases, additional deflecting pins and screws were used, held near the nail. The blocking of the rod was carried out in a standard way with the 
introduction of 1-2 screws into each of the fragments.
B-th B., 35 years old, closed fracture of the bones of the right tibia with displacement. Intramedullary osteosynthesis was performed in a planned manner (Fig. 1).
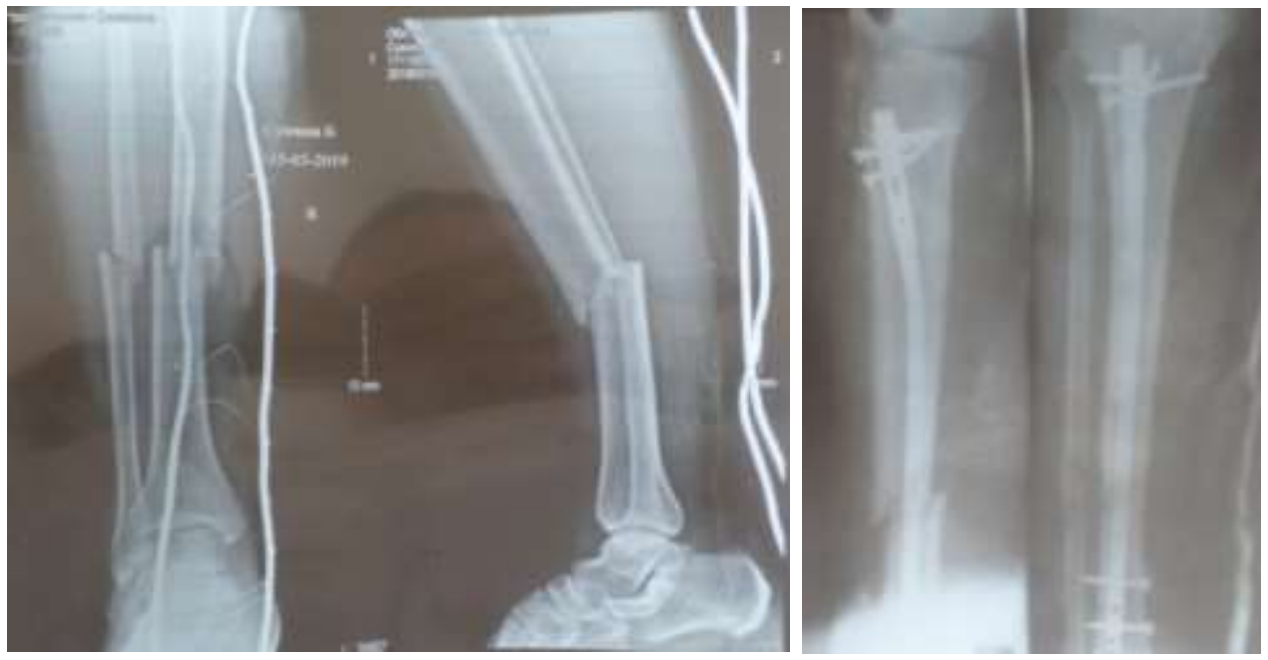

Figure: 1. X-ray of the bones of the right leg before and after the BIOS. B-th A., 50 years old, open fracture of the bones of the left leg s / c with displacement. Intramedullary osteosynthesis was performed in a planned manner (Fig. 2).

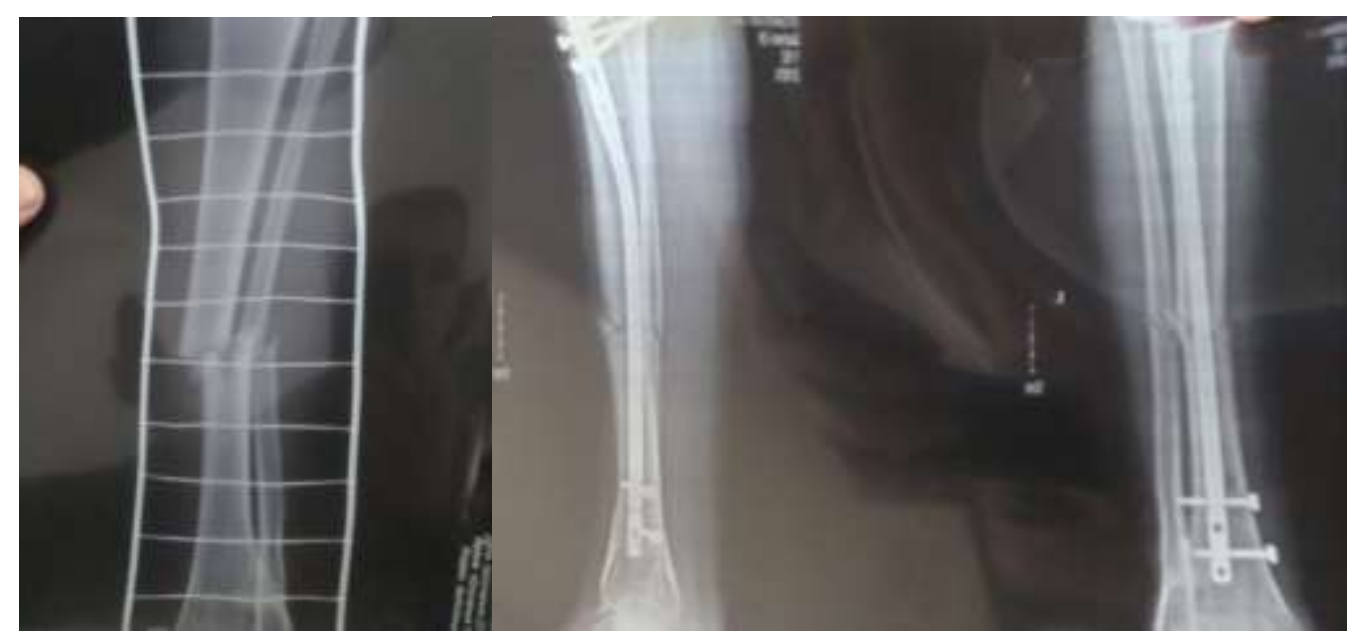

Figure: 2. X-ray of the bones of the left leg before and after the BIOS. 
When studying long-term results, clinical and radiological data were evaluated. To systematize and objectify clinical results, the developed 100-point assessment scheme, including the determination of the range of motion, axial deviations, the state of soft tissues, the severity of pain and edema, the degree and timing of recovery.

\section{RESULTS}

When studying long-term results, clinical and radiological data were evaluated. To systematize and objectify clinical results, a developed 100-point assessment scheme was used, including the determination of the range of motion, axial deviations, the state of soft tissues, the severity of pain and edema, the degree and timing of recovery. The timing of the consolidation of fractures. In the majority of patients with fractures of the middle and lower third of the lower leg, rapid consolidation of fractures was observed by 3-4 months after surgery with the transition to full load of the limb with body weight. In 7 (14\%) of patients, clear signs of fracture consolidation were radiologically determined by 6 months after surgery, in 4 ( $8 \%)$ - by 8 months.

\section{CONCLUSIONS}

1. Intramedullary blocking osteosynthesis is reliable and a predictable method of treating diaphyseal fractures of the tibia, which allows to achieve restoration of limb function in the shortest possible time in most patients.

2. Intramedullary osteosynthesis of diaphyseal fractures of the upper third of the lower leg is associated with a number of technical difficulties and must be performed by experienced surgeons. To treat such injuries, fixators with extended blocking capabilities are required.
3. Control of the postoperative course of consolidation from the side the operating surgeon is optimal both for reducing the time of treatment of patients and for timely correction of surgical tactics.

\section{REFERENCES}

1. Vasin I.V., Pisarev V.V., Lvov S.E. Surgical treatment of tibial fractures using a device for intramedullary osteosynthesis of the tibia with screw locking / Modern problems of science and education, 2012. No. 6: 43-89.

2. Gaiko G., Kalashnikov A.V., Vdovichenko K.V., Chalaidyuk T.P. Analysis of the results of treatment of diaphyseal fractures of the tibia using various types of osteosynthesis. Osteosynthesis, 2012-3 (20): 1620.

3. Klimovitskiy V.G., Oksimets V.M. Symposium "Fractures, but they have not grown up, melting pseudoarthrosis." Trauma, 2012.13 (4): 166-174.

4. Sergeev S.V. Modern fracture treatment technologies. Osteosynthesis. Referrer. zhurn., 2012.2 (15): 9-13.

5. Calori G.M., Giannoudis P.V. Enhancement of fracture healing with the diamond concept: the role of the biological chamber. Injury 2011.42 (11): 1191.

6. Fong K., Truong V., Foote C.J. et al. Predictors of nonunion and reoperation of the tibia: an observational study. BMC Musculoskelet. Disord., 2013.14: 103.

7. Zimmermann G., Moghaddam A. Trauma: Non-Union: New Trends. In: European Instructional Lectures. 11th EFORT Congress, 24 Mar., Madrid, Spain, 2010-10: 15-19. 